\title{
Pacific
}

Journal of

Mathematics

\section{MILNOR OPEN BOOKS OF LINKS \\ OF SOME RATIONAL SURFACE SINGULARITIES}

MoHan Bhupal AND BuraK OzBagCI

Volume 254 No. 1

November 2011 


\title{
MILNOR OPEN BOOKS OF LINKS OF SOME RATIONAL SURFACE SINGULARITIES
}

\author{
MOHAN BHUPAL AND BURAK OZBAGCI
}

\begin{abstract}
We determine Legendrian surgery diagrams for the canonical contact structures of links of rational surface singularities that are also small Seifert fibered 3-manifolds. Moreover, we describe an infinite family of Milnor fillable contact 3-manifolds so that, for each member of this family, the Milnor genus and Milnor norm are strictly greater than the support genus and support norm of the canonical contact structure. For some of these contact structures we construct supporting Milnor open books.
\end{abstract}

\section{Introduction}

The link of a normal complex surface singularity carries a canonical contact structure $\xi_{\text {can }}$ (also known as the Milnor fillable contact structure) which is supported by any Milnor open book on this link [Caubel et al. 2006]. The canonical contact structure $\xi_{\text {can }}$ is known to be Stein fillable [Bogomolov and de Oliveira 1997] and therefore it is tight [Eliashberg and Gromov 1991]. In fact, $\xi_{\text {can }}$ is universally tight, that is, the pullback to the universal cover is tight [Lekili and Ozbagci 2010].

Etnyre and Ozbagci [2008] defined three numerical invariants of contact structures in terms of open books supporting the contact structures. These invariants are the support genus $\operatorname{sg}(\xi)$ (the minimal genus of a page of a supporting open book for $\xi$ ), the binding number $b n(\xi)$ (the minimal number of binding components of a supporting open book for $\xi$ with minimal genus pages) and the support norm $\operatorname{sn}(\xi)$ (minus the maximal Euler characteristic of a page of a supporting open book for $\xi)$.

Altınok and Bhupal [2008] derived a new set of invariants specifically for the canonical contact structure $\xi_{\text {can }}$ on the link of a complex surface singularity by restricting the set of open books to only Milnor open books on the link at hand. In this article we will call these invariants the Milnor genus $\operatorname{Mg}\left(\xi_{\text {can }}\right)$, the Milnor

Ozbagci was partially supported by the 107T053 and BIDEP-2219 research grants of the Scientific and Technological Research Council of Turkey and the Marie Curie International Outgoing Fellowship 236639.

MSC2010: 32S25, 32S55, 53D10, 57R17.

Keywords: contact structure, Milnor open book, support genus, surface singularity, Milnor fillable,

Milnor genus. 
binding number $\operatorname{Mb}\left(\xi_{\text {can }}\right)$, and the Milnor norm $\operatorname{Mn}\left(\xi_{\text {can }}\right)$ of the canonical contact structure $\xi_{\text {can }}$. The well-known Milnor number corresponds to the first Betti number of the page of the Milnor open book in our context.

It follows by definition that $\operatorname{sg}\left(\xi_{\text {can }}\right) \leq \operatorname{Mg}\left(\xi_{\text {can }}\right)$ and $\operatorname{sn}\left(\xi_{\text {can }}\right) \leq \operatorname{Mn}\left(\xi_{\text {can }}\right)$, since the set of Milnor open books is a subset of all open books on the link of a surface singularity. (No such inequality exists in general, however, between bn $\left(\xi_{\text {can }}\right)$ and $\mathrm{Mb}\left(\xi_{\mathrm{can}}\right)$.) In Section 9, we show that for each positive integer $k$, there is a rational surface singularity whose canonical contact structure $\xi_{\text {can }}$ satisfies

$$
k \leq \operatorname{Mg}\left(\xi_{\mathrm{can}}\right)-\operatorname{sg}\left(\xi_{\mathrm{can}}\right) \text { and } k \leq \operatorname{Mn}\left(\xi_{\mathrm{can}}\right)-\operatorname{sn}\left(\xi_{\mathrm{can}}\right) .
$$

An immediate consequence is the existence of links of surface singularities carrying open books that are not isomorphic to Milnor open books. As another consequence, we deduce that Milnor open books are neither norm- nor genus-minimizing, although our aim originally was to show that the support genus of a Milnor fillable contact structure is realized by a Milnor open book. We find this result interesting since there are other instances in geometric topology where the "complex representatives" are minimizers. Most notably, the link of a complex plane curve singularity bounds a smooth complex curve of genus equal to its Seifert genus.

The aforementioned examples are the canonical contact structures on links of some rational surface singularities which are also small Seifert fibered 3-manifolds. In Section 8, we identify the canonical contact structures on all such manifolds via their Legendrian surgery diagrams. A Legendrian surgery diagram is perhaps the most efficient way of describing a contact structure from a topological point of view, since it also allows one to calculate many invariants of the contact structure (for example, the Euler class of the underlying oriented plane field) by easily converting the diagram into a smooth handlebody diagram.

\section{Open books and contact structures}

A complete exposition of the correspondence between open books and contact structures can be found in the lecture notes of Etnyre [2006]. In this section, we recall some basic definitions.

Suppose that for an oriented link $B$ in a closed and oriented 3-manifold $Y$, the complement $Y \backslash B$ fibers over the circle as $p: Y \backslash B \rightarrow S^{1}$ such that $p^{-1}(t)=\Sigma_{t}$ is the interior of a compact surface with $\partial \Sigma_{t}=B$ for all $t \in S^{1}$. Then $(B, p)$ is called an open book decomposition (or just an open book) of $Y$. For each $t \in S^{1}$, the surface $\Sigma_{t}$ is called a page, while $B$ is referred to as the binding of the open book.

The monodromy of the fibration $p$ is defined as the diffeomorphism of a fixed page which is given by the first return map of a flow that is transverse to the 
pages and meridional near the binding. The isotopy class of this diffeomorphism is independent of the chosen flow and is called the monodromy of the open book decomposition. In order to describe the monodromy of an open book explicitly, one usually writes it as a product of Dehn twists along some curves on the page. In this paper, we will denote a right-handed (respectively left-handed) Dehn twist along a curve $\alpha$ as $\alpha$ (respectively $\alpha^{-1}$ ), for simplicity.

An open book can also be described as follows. First consider the mapping torus

$$
\Sigma_{\phi}=([0,1] \times \Sigma) /((1, x) \sim(0, \phi(x))),
$$

where $\Sigma$ is a compact oriented surface with $r$ boundary components and $\phi$ is an element of the mapping class group $\Gamma_{\Sigma}$ of $\Sigma$. Since $\phi$ is the identity map on $\partial \Sigma$, the boundary $\partial \Sigma_{\phi}$ of the mapping torus $\Sigma_{\phi}$ can be canonically identified with $r$ copies of $T^{2}=S^{1} \times S^{1}$, where the first $S^{1}$ factor is identified with [0,1]/(0 1) and the second one comes from a component of $\partial \Sigma$. Now we glue in $r$ copies of $D^{2} \times S^{1}$ to cap off $\Sigma_{\phi}$ so that $\partial D^{2}$ is identified with $S^{1}=[0,1] /(0 \sim 1)$ and the $S^{1}$ factor in $D^{2} \times S^{1}$ is identified with a boundary component of $\partial \Sigma$. Thus we get a closed 3-manifold $Y=\Sigma_{\phi} \cup_{r} D^{2} \times S^{1}$ equipped with an open book decomposition whose binding is the union of the core circles of the copies of $D^{2} \times S^{1}$ that we glue to $\Sigma_{\phi}$ to obtain $Y$. In conclusion, an element $\phi \in \Gamma_{\Sigma}$ determines a 3-manifold together with an abstract open book decomposition on it. By conjugating the monodromy $\phi$ of an open book on a 3-manifold $Y$ by an element in $\Gamma_{\Sigma}$, we get an isomorphic open book on a 3-manifold $Y^{\prime}$ which is diffeomorphic to $Y$.

It has been known for a long time that every closed and oriented 3-manifold admits an open book decomposition. A new interest in open books on 3-manifolds arose recently from their connection to contact structures, which we will describe very briefly.

Recall that a (positive) contact structure $\xi$ on an oriented 3-manifold is locally the kernel of a 1 -form $\alpha$ such that $\alpha \wedge d \alpha>0$. In this paper we assume $\xi$ is coorientable, that is, $\alpha$ is a global 1 -form.

Definition 2.1. An open book decomposition $(B, p)$ of a 3-manifold $Y$ is said to support a contact structure $\xi$ on $Y$ if $\xi$ can be represented by a contact form $\alpha$ such that $\alpha$ evaluates positively on $B$ and $d \alpha$ is a symplectic form on every page.

Thurston and Winkelnkemper [1975] associated a contact structure to every open book. This contact structure is in fact supported by the underlying open book. (Definition 2.1 was not available at the time.) To state the converse we need a little digression.

Suppose that an open book decomposition with page $\Sigma$ is specified by $\phi \in \Gamma_{\Sigma}$. Attach a 1-handle to the surface $\Sigma$ connecting two points on $\partial \Sigma$ to obtain a new surface $\Sigma^{\prime}$. Let $\gamma$ be a closed curve in $\Sigma^{\prime}$ going over the new 1-handle exactly 
once. Define a new open book decomposition with

$$
\phi^{\prime}=\phi \circ t_{\gamma} \in \Gamma_{\Sigma^{\prime}},
$$

where $t_{\gamma}$ denotes the right-handed Dehn twist along $\gamma$. The resulting open book decomposition is called a positive stabilization of the one defined by $\phi$. Although the resulting monodromy depends on the chosen curve $\gamma$, the 3-manifold specified by $\left(\Sigma^{\prime}, \phi^{\prime}\right)$ is diffeomorphic to the 3-manifold specified by $(\Sigma, \phi)$. A converse to Thurston and Winkelnkemper's result is:

Theorem 2.2 [Giroux 2002]. Every contact structure on a 3-manifold is supported by an open book. Two open books supporting the same contact structure admit a common positive stabilization. Moreover two contact structures supported by the same open book are isotopic.

\section{Legendrian surgery diagrams}

Recall that a knot in a contact 3-manifold is called Legendrian if it is everywhere tangent to the contact planes. In order to have a better understanding of the topological constructions in the later sections, we discuss a standard way to visualize Legendrian knots in $\mathbb{R}^{3}$ (and therefore $S^{3}$ ) equipped with the standard contact structure $\xi_{\mathrm{st}}=\operatorname{ker}(d z+x d y)$.

Now consider a Legendrian knot $L \subset\left(\mathbb{R}^{3}, \xi_{\mathrm{st}}\right)$ and take its front projection, that is, its projection to the $y z$-plane. This projection has no vertical tangencies since $-d z / d y=x \neq \infty$, and for the same reason, at a crossing the strand with smaller slope is in front. It turns out that $L$ can be $C^{2}$-approximated by a Legendrian knot for which the projection has only transverse double points and cusp singularities (see [Geiges 2008], for example). Conversely, a knot projection with these properties gives rise to a unique Legendrian knot in $\left(\mathbb{R}^{3}, \xi_{\mathrm{st}}\right)$ by defining $x$ from the projection as $-d z / d y$. Since any projection can be isotoped to satisfy the above properties, every knot in $S^{3}$ can be isotoped (nonuniquely) to a Legendrian knot.

Two classical invariants of a Legendrian knot $L$ are the Thurston-Bennequin number $\operatorname{tb}(L)$ and the rotation number $\operatorname{rot}(L)$. Recall that $\operatorname{tb}(L)$ is the contact framing of $L$ (measured with respect to the Seifert framing in $S^{3}$ ), which can be easily computed from a front projection of $L$. Define the writhe $w(L)$ of $L$ as the sum of signs of the double points. For this to make sense, we need to fix an orientation on the knot, but the result is independent of this choice. If $c(L)$ is the number of cusps, then $\operatorname{tb}(L)=w(L)-\frac{1}{2} c(L)$.

The rotation number $\operatorname{rot}(L)$ is defined by trivializing $\xi_{\text {st }}$ along a Seifert surface $\Sigma$ whose oriented boundary is $L$ and then taking the winding number of $T L$ with respect to this trivialization. For this invariant to make sense we need to orient $L$, and the result changes sign by reversing the orientation. Since $H^{2}\left(S^{3} ; \mathbb{Z}\right)=0$, this 
number is independent of the chosen trivialization. If $c_{d}(L)$ and $c_{u}(L)$ denote the number of down and up cusps in the projection, then $\operatorname{rot}(L)=\frac{1}{2}\left(c_{d}(L)-c_{u}(L)\right)$.

To describe the Stein fillable contact structures dealt with in this paper, we use Legendrian knots (actually their front projections) as follows: Consider the standard Stein 4-ball $B^{4}$ with the induced standard contact structure on its boundary. Then attach Weinstein 2-handles [1991] along an arbitrary Legendrian link in $\partial B^{4}=S^{3}$ to this ball. By [Eliashberg 1990], the Stein structure on $B^{4}$ extends over the 2-handles as long as the attaching framing of each 2-handle is one less than the Thurston-Bennequin number. The resulting Stein domain has an induced contact structure on its boundary which can be represented by the front projection of the Legendrian link along which we attach the 2-handles. Such a front projection is called a Legendrian surgery diagram (see [Gompf 1998] for a thorough discussion). Legendrian surgery is equivalent to performing contact (-1)-surgery along the given Legendrian link in the standard contact $S^{3}$ [Ding and Geiges 2004]. To describe all Stein fillable contact structures in general, one needs 1-handles as well, but those will not appear in our discussion.

\section{Milnor open books and canonical contact structures}

Let $(X, x)$ be an isolated normal complex surface singularity (see [Némethi 1999]). Fix a local embedding of $(X, x)$ in $\left(\mathbb{C}^{N}, 0\right)$. Then a small sphere $S_{\epsilon}^{2 N-1} \subset \mathbb{C}^{N}$ centered at the origin intersects $X$ transversely, and the complex hyperplane distribution $\xi_{\text {can }}$ on $M=X \cap S_{\epsilon}^{2 N-1}$ induced by the complex structure on $X$ is called the canonical contact structure. It is known that, for sufficiently small radius $\epsilon$, the contact manifold is independent of $\epsilon$ and the embedding, up to isomorphism. The 3-manifold $M$ is called the link of the singularity and $\left(M, \xi_{\text {can }}\right)$ is called the contact boundary of $(X, x)$. While $Y$ denotes a general 3-manifold, we use $M$ for those which are Milnor-filled.

Definition 4.1. A contact manifold $(Y, \xi)$ is said to be Milnor fillable and the germ $(X, x)$ is called a Milnor filling of $(Y, \xi)$ if $(Y, \xi)$ is isomorphic to the contact boundary $\left(M, \xi_{\text {can }}\right)$ of some isolated complex surface singularity $(X, x)$. In addition, we say that a closed and oriented 3-manifold $Y$ is Milnor fillable if it carries a contact structure $\xi$ so that $(Y, \xi)$ is Milnor fillable. Such a contact structure $\xi$ is called a Milnor fillable contact structure.

By a theorem in [Mumford 1961], if a contact 3-manifold is Milnor fillable, then it can be obtained by plumbing oriented circle bundles over surfaces according to a weighted graph with negative definite intersection matrix. Conversely, it follows from a well-known theorem of [Grauert 1962] that any 3-manifold that is given by plumbing oriented circle bundles over surfaces according to a weighted graph 
with negative definite intersection matrix is Milnor fillable. As for the uniqueness of Milnor fillable contact structures, we have the fundamental result:

Theorem 4.2 [Caubel et al. 2006]. Any closed and oriented 3-manifold has at most one Milnor fillable contact structure up to isomorphism.

In summary, Milnor fillability of a closed and oriented 3-manifold $Y$ is determined entirely by its topology and if $Y$ is Milnor fillable, then it carries a canonical contact structure $\xi_{\text {can }}$ which is unique up to isomorphism.

Since the groundbreaking result (Theorem 2.2) of [Giroux 2002], the geometry of contact structures is often studied via their topological counterparts, namely open book decompositions. In the realm of surface singularities this fits nicely with [Milnor 1968].

Definition 4.3. Given an analytic function $f:(X, x) \rightarrow(\mathbb{C}, 0)$ vanishing at $x$, with an isolated singularity at $x$, the open book decomposition $\mathscr{O}_{B}$ of the link $M$ of $(X, x)$ with binding $L=M \cap f^{-1}(0)$ and projection $\pi=f /|f|: M \backslash L \rightarrow S^{1} \subset \mathbb{C}$ is called the Milnor open book induced by $f$.

Such functions $f$ exist and one can talk about many Milnor open books on the singularity link $M$. Therefore, there are many Milnor open books on any given Milnor fillable contact 3-manifold $(Y, \xi)$, since, by definition, it is isomorphic to the link $\left(M, \xi_{\text {can }}\right)$ of some isolated complex surface singularity $(X, x)$.

A Milnor open book on a Milnor fillable 3-manifold $Y$ has two essential features as shown in [Caubel et al. 2006]:

(i) It supports the canonical contact structure $\xi_{\text {can }}$.

(ii) It is horizontal when considered on the plumbing description of $Y$.

Suppose that the 3-manifold $Y$ is obtained by plumbing oriented circle bundles $M_{i} \rightarrow S_{i}$, for $i=1,2, \ldots, r$. For any $r$-tuple of nonnegative integers $\underline{n}=$ $\left(n_{1}, n_{2}, \ldots, n_{r}\right)$, a vertical link of type $\underline{n}$ consists of a disjoint union of $n_{i}$ generic fibers from each bundle $M_{i} \rightarrow S_{i}$. An open book on $Y$ is called horizontal if its binding is a vertical link and its pages are transverse to the fibers. We also require that the orientation induced on the binding by the pages coincides with the orientation of the fibers induced by the fibration.

\section{Rational surface singularities}

Let $(X, x)$ be a germ of a normal complex surface having a singularity at $x$. Recall that $(X, x)$ is called rational [Artin 1966] if the geometric genus $p_{g}:=$ $\operatorname{dim}_{\mathbb{C}} H^{1}\left(\widetilde{X}, \mathcal{O}_{\tilde{X}}\right)$ is equal to zero, where $\widetilde{X} \rightarrow X$ is a resolution of the singular point $x \in X$. This definition does not depend on the resolution.

Now fix a resolution $\pi: \widetilde{X} \rightarrow X$ and denote the irreducible components of the exceptional divisor $E=\pi^{-1}(x)$ by $\bigcup_{i=1}^{r} E_{i}$. The fundamental cycle of $E$ is 
by definition the componentwise smallest nonzero effective divisor $Z=\sum z_{i} E_{i}$ satisfying $Z \cdot E_{i} \leq 0$ for all $1 \leq i \leq r$.

The singularity $(X, x)$ is rational if each irreducible component $E_{i}$ of the exceptional divisor $E$ is isomorphic to $\mathbb{C} P^{1}$ and

$$
Z \cdot Z+\sum_{i=1}^{r} z_{i}\left(-E_{i}^{2}-2\right)=-2,
$$

where $Z=\sum z_{i} E_{i}$ is the fundamental cycle of $E$. Once a dual resolution graph of a surface singularity is given, then the so-called Laufer algorithm [1972] can be applied to calculate the corresponding fundamental cycle. Therefore the criterion in the equation above makes it particularly simple to identify the given singularity as rational.

Suppose that $(X, x)$ is a germ of a normal complex surface having a rational singularity at $x$.

Theorem 5.1 [Altınok and Bhupal 2008]. Both the page-genus and the page-genus plus the number of binding components of the Milnor open book $\mathrm{O}_{f}$ are minimized when $f$ is taken to be the restriction of a generic linear form on $\mathbb{C}^{N}$ to $(X, x)$ for some local embedding of $(X, x)$ in $\left(\mathbb{C}^{N}, 0\right)$.

If $\mathscr{O S}_{\min }$ denotes the Milnor open book given by taking the restriction of a generic linear form on $\mathbb{C}^{N}$ to $(X, x)$ for some local embedding of $(X, x)$ in $\left(\mathbb{C}^{N}, 0\right)$, then Theorem 5.1 implies that $\operatorname{Mg}\left(\xi_{\text {can }}\right)=g\left(\mathscr{O S}_{\min }\right)$ and $\operatorname{Mb}\left(\xi_{\text {can }}\right)=b c\left(\mathscr{O} \mathscr{B}_{\min }\right)$, where $g(\mathscr{O B})$ (respectively bc(OB)) denotes the page-genus (respectively the number of binding components) of the open book $\mathscr{O P B}$. We will call $\mathscr{O O}_{\min }$ the minimal Milnor open book. For the Milnor norm, from the definition,

$$
\operatorname{Mn}\left(\xi_{\text {can }}\right)=\min (2 g(\mathscr{O} \mathscr{B})-2+\operatorname{bc}(\mathscr{O})),
$$

where the minimum is taken over all supporting Milnor open books $\mathscr{O} \mathscr{B}$. Hence it also follows from Theorem 5.1 that

$$
\operatorname{Mn}\left(\xi_{\text {can }}\right)=2 g\left(\mathscr{O P}_{\min }\right)-2+\mathrm{bc}\left(\mathscr{O P}_{\min }\right)=2 \mathrm{Mg}\left(\xi_{\mathrm{can}}\right)-2+\mathrm{Mb}\left(\xi_{\mathrm{can}}\right) .
$$

Remark 5.2. The equation $\operatorname{sn}(\xi)=2 \operatorname{sg}(\xi)-2+\operatorname{bn}(\xi)$ is not necessarily true for an arbitrary contact structure $\xi$, as illustrated in [Baldwin and Etnyre 2011; Etgü and Lekili 2010].

Suppose that $\pi: \widetilde{X} \rightarrow X$ is a good resolution of $(X, x)$ and let $E_{1}, \ldots, E_{r}$ denote the irreducible components of the exceptional divisor $E$. Given an analytic function $f:(X, x) \rightarrow(\mathbb{C}, 0)$ vanishing at $x$, with an isolated singularity at $x$, the open book decomposition $\mathscr{O}_{f}$ is a horizontal open book with binding a vertical link of type $\underline{n}=\left(n_{1}, \ldots, n_{r}\right)$, where the $n_{i}$ are defined as follows: Consider the decomposition $(f \circ \pi)=(f \circ \pi)_{e}+(f \circ \pi)_{s}$ of the divisor $(f \circ \pi) \in \operatorname{Div}(\tilde{X})$ into its exceptional 
and strict parts such that $(f \circ \pi)_{e}$ is supported on $E$ and $\operatorname{dim}\left(\left|(f \circ \pi)_{s} \cap E\right|\right)<1$. Then $n_{i}$ is the number of components of $(f \circ \pi)_{s}$ which cut $E_{i}$. It is known that the $r$-tuple $\underline{n}=\left(n_{1}, \ldots, n_{r}\right)$ satisfies

$$
I(\Gamma(\pi)) \underline{m}^{t}=-\underline{n}^{t}
$$

for some $r$-tuple $\underline{m}=\left(m_{1}, \ldots, m_{r}\right)$ of positive integers, where $I(\Gamma(\pi))$ denotes the intersection matrix of the dual resolution graph $\Gamma(\pi)$ associated to $\pi$ and $t$ is used for transpose. In [Altınok and Bhupal 2008, Lemma 3.1] it is proved that

$$
g\left(\mathscr{O} \mathscr{B}_{f}\right)=1+\sum_{i=1}^{r} \frac{\left(v_{i}-2\right) m_{i}+\left(m_{i}-1\right) n_{i}}{2},
$$

where $v_{i}$ denotes the number of irreducible curves $E_{j}, j \neq i$, in $E$ intersecting $E_{i}$ for $i=1, \ldots, r$. Also

$$
\operatorname{bc}\left(\mathscr{O} \mathscr{B}_{f}\right)=\sum_{i=1}^{r} n_{i} .
$$

On the other hand, it follows from [Artin 1966] that for any $r$-tuple $\underline{n}$ of nonnegative integers which satisfies (5-1) for some $r$-tuple $\underline{m}$ of positive integers there is a Milnor open book decomposition of the boundary of $(X, x)$ whose binding is equivalent to a vertical link of type $\underline{n}$.

The upshot is that if $Z=\sum_{i=1}^{r} z_{i} E_{i}$ is the fundamental cycle of the resolution $\pi$, then the above construction for the $r$-tuple $\underline{m}=\left(z_{1}, \ldots, z_{r}\right)$ gives the minimal Milnor open book $\mathscr{O}_{\min }$, which we will also denote by $\mathscr{O} \mathscr{B}(\underline{m})$.

Remark 5.3. Némethi and Tosun [2011] (see also [Némethi 2008]) give a generalization of Theorem 5.1 for all Milnor fillable rational homology 3-spheres and prove

$$
\operatorname{Mg}\left(\xi_{\text {can }}\right)=Z \cdot E-Z \cdot Z \text { and } \operatorname{Mb}\left(\xi_{\text {can }}\right)=-Z \cdot E
$$

\section{Tight contact structures on small Seifert fibered 3-manifolds}

A small Seifert fibered 3-manifold $Y$ is a closed and oriented 3-manifold which admits a Seifert fibration over $S^{2}$ with at most three singular fibers. Equivalently, such a manifold $Y=Y\left(e_{0} ; r_{1}, r_{2}, r_{3}\right)$ can be described by the rational surgery diagram depicted in Figure 1, where $e_{0} \in \mathbb{Z}$ and $r_{i} \in(0,1) \cap \mathbb{Q}$, for $i=1,2,3$.

One can also obtain an integral surgery description of $Y$ as follows. Consider the continued fraction expansion of $-1 / r_{i}$ :

$$
-\frac{1}{r_{i}}=a_{1}^{(i)}-\frac{1}{a_{2}^{(i)}-\frac{1}{\ddots \cdot-\frac{1}{a_{n_{i}}^{(i)}}}}, \quad i=1,2,3,
$$




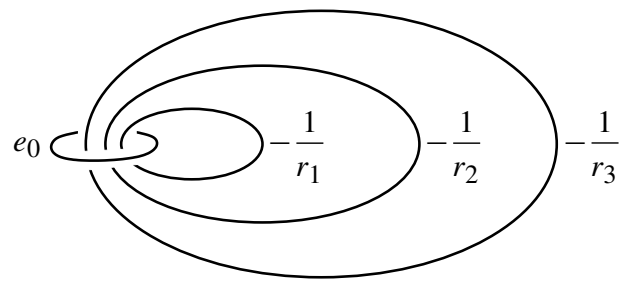

Figure 1. Rational surgery diagram for the small Seifert fibered 3-manifold $Y\left(e_{0} ; r_{1}, r_{2}, r_{3}\right)$.

for some uniquely determined integers $a_{1}^{(i)}, \ldots, a_{n_{i}}^{(i)} \leq-2$. Let $K_{0}^{(i)}$ denote the unknot with framing $e_{0}$ in Figure 1, for $i=1,2,3$. Now replace the unknot with coefficient $-1 / r_{i}$, by a chain of unknots $K_{1}^{(i)}, \ldots, K_{n_{i}}^{(i)}$ with integral framings $a_{1}^{(i)}, \ldots, a_{n_{i}}^{(i)}$, respectively, so that

$$
\operatorname{lk}\left(K_{j}^{(i)}, K_{k}^{(i)}\right)= \begin{cases} \pm 1 & \text { if }|j-k|=1 \\ 0 & \text { otherwise, }\end{cases}
$$

for $0 \leq j, k \leq n_{i}$ and $i=1,2,3$. As discussed in the next paragraph, this "starshaped" integral surgery presentation is very convenient in terms of describing Stein fillable contact structures on $Y$.

Wu [2006] classified all tight contact structures on $Y$ up to isotopy under the assumption that $e_{0} \leq-3$. They are all Stein fillable and can be represented by Legendrian surgery diagrams which are obtained by all possible Legendrian realizations (without double points) of the unknots imposed by the surgery coefficients in the integral surgery description of $Y$. Moreover, Ghiggini [2008] showed that the same classification scheme works for the case $e_{0}=-2$, as long as $Y$ is assumed to be an $L$-space (a rational homology sphere whose Heegaard Floer homology is as simple as possible, that is, $\left.\operatorname{rk} \widehat{H F}(Y)=\left|H_{1}(Y ; \mathbb{Z})\right|\right)$.

The link of any rational surface singularity is an $L$-space by a theorem in [Némethi 2005]. A necessary condition for the 3-manifold $Y=Y\left(e_{0} ; r_{1}, r_{2}, r_{3}\right)$ to be the link of a rational singularity is that $e_{0} \leq-2$; however, it is not sufficient.

\section{Planar Milnor open books}

By a weighted plumbing graph we mean a graph such that each vertex is decorated by some integer "weight". Such a graph naturally represents a closed oriented 3manifold called a graph manifold, which can be described as follows. For each vertex of the graph, take an oriented circle bundle over $S^{2}$ whose Euler number is equal to the weight of that vertex and plumb these bundles together according to the given graph. In other words, if there is an edge connecting two vertices in $\Gamma$, then plumb the circle bundles corresponding to these vertices. More precisely, first remove a neighborhood of a circle fiber on each circle bundle which is given by the 
preimage of a disk on the base sphere. The resulting boundary torus on each circle bundle can be identified with $S^{1} \times S^{1}$ using the natural trivialization of the circle fibration over the disk that is removed. Now glue these bundles together using the diffeomorphism that exchanges the two circle factors on the boundary tori.

If the graph is a tree, in particular, then an integral surgery presentation of the 3-manifold is readily available by replacing each vertex by an unknot framed by the weight of that vertex such that any two of these unknots are linked once if there is an edge between the vertices they represent and they are unlinked otherwise.

Recall that the degree of a vertex in a graph is the number of edges emanating from that vertex. A vertex in a weighted plumbing graph is called a bad vertex if the sum of the weight (the Euler number) and the degree of that vertex is positive.

Proposition 7.1. If $Y$ is the link of a rational surface singularity presented by a plumbing tree without any bad vertices, then $Y$ carries a planar Milnor open book.

Proof. Let $\Gamma$ be a plumbing tree for $Y$ with $r$ vertices $v_{1}, \ldots, v_{r}$ so that $v_{i}$ has Euler number $e_{i}$ and degree $d_{i}$. Suppose that $\Gamma$ has no bad vertices, that is, $e_{i}+d_{i} \leq 0$ for $i=1, \ldots, r$. Etgü and Ozbagci [2006] constructed an explicit planar horizontal open book $O \mathscr{B}$ with binding a vertical link of type

$$
\underline{n}=\left(-e_{1}-d_{1},-e_{2}-d_{2}, \ldots,-e_{r}-d_{r}\right)
$$

on such a graph manifold $Y$. It is easy to check that for $\underline{m}=(1,1, \ldots, 1)$, we have

$$
I(\Gamma) \underline{m}^{t}=-\underline{n}^{t},
$$

where $I(\Gamma)$ denotes the intersection matrix of the tree $\Gamma$ which defines $Y$. Suppose that $Y$ is the link of some rational surface singularity $(X, x)$. Then $\underline{m}$ corresponds to the fundamental cycle of the minimal resolution of $(X, x)$. As we indicated in the paragraph preceding Remark 5.3, the binding of the minimal Milnor open book $O \mathscr{S}_{\min }$ is a vertical link of type $\underline{n}$ as well. Since the open books $\mathscr{O} \mathscr{B}$ and $\mathrm{OP}_{\min }$ on the rational homology 3-sphere $Y$ have equivalent bindings, by [Caubel and Popescu-Pampu 2004] it follows that $\mathscr{O} \mathscr{B}$ is isotopic to $\mathscr{O B}_{\min }$. This proves that $Y$ carries a planar Milnor open book, that is, $\operatorname{Mg}\left(\xi_{\text {can }}\right)=0$. Moreover, since the binding of $\mathscr{O} \mathscr{B}$ is a vertical link of type $\underline{n}$, we have $\mathrm{Mb}\left(\xi_{\text {can }}\right)=-\sum_{i=1}^{r}\left(e_{i}+d_{i}\right)$ and hence $\operatorname{Mn}\left(\xi_{\text {can }}\right)=\operatorname{Mb}\left(\xi_{\text {can }}\right)-2$.

In the proof of Proposition 7.1 we showed that the horizontal open book $O_{B}$ constructed in [Etgü and Ozbagci 2006] is isotopic to a Milnor open book, which implies that the horizontal contact structure supported by $\mathscr{O} \mathscr{B}$ is isomorphic to $\xi_{\text {can }}$ on such rational singularity links. On the other hand, Legendrian surgery diagrams of such horizontal contact structures (which are known to be Stein fillable) were studied in [Ozbagci 2008]. A lens space $L(p, q)$, for example, is given by a linear plumbing diagram without any bad vertices. Equivalently, an integral surgery 
diagram of $L(p, q)$ is given by a chain of unknots so that the linking number between every two consecutive unknots is equal to \pm 1 and the framing of every unknot is less than or equal to -2 .

Proposition 7.2 [Ozbagci 2008, Proposition 3.2]. Orient the unknots in the linear integral surgery diagram giving $L(p, q)$ so that the linking number is +1 between any two consecutive unknots. A Legendrian surgery diagram for $\xi_{\mathrm{can}}$ on $L(p, q)$ is obtained by Legendrian realizing each unknot with maximum possible rotation number imposed by its surgery coefficient.

A Legendrian realization of an unknot with maximum possible rotation number imposed by its surgery coefficient is given by a front projection without any double points and with a single up cusp or a single down cusp, depending on the chosen orientation. Once we orient any knot in the chain describing $L(p, q)$, the orientations of the other knots are determined uniquely, by the hypothesis in Proposition 7.2. Hence there are two choices of overall orientations inducing two Legendrian surgery diagrams for $\xi_{\text {can }}$ which are mirror images of each other. In other words, $\xi_{\text {can }}$ is represented by a Legendrian surgery diagram where all the zigzags of all the Legendrian unknots are on the left or all on the right (see [Ozbagci 2008, Figure 4]). Nevertheless, these two diagrams induce isomorphic contact structures, where the underlying plane fields are obtained from each other by simply reversing the orientations.

There are two key properties used to prove Proposition 7.2: (i) the linear plumbing diagram of $L(p, q)$ does not have any bad vertices and (ii) all the tight contact structures on $L(p, q)$ are Stein fillable and given by all possible Legendrian realizations (without double points) of the unknots in the plumbing diagram. In Section 6, we described a star-shaped plumbing diagram of a small Seifert fibered 3-manifold $Y=Y\left(e_{0} ; r_{1}, r_{2}, r_{3}\right)$ which does not include any bad vertices as long as $e_{0} \leq-3$. Therefore it is straightforward to generalize Proposition 7.2 to all rational singularity links which are small Seifert fibered spaces with $e_{0} \leq-3$, using the methods in [Ozbagci 2008] coupled with Wu's classification [2006] of tight contact structures on such manifolds. This generalization is included in the statement of Theorem 8.1 for which we present a more conceptual proof.

\section{Legendrian surgery diagrams for canonical contact structures}

Theorem 8.1. Let $Y=Y\left(e_{0} ; r_{1}, r_{2}, r_{3}\right)$ be a small Seifert fibered 3-manifold that is diffeomorphic to the link of some rational surface singularity. Orient the unknots in the star-shaped integral surgery diagram giving $Y$, so that the linking number is +1 between any two consecutive unknots in every chain. A Legendrian surgery diagram for $\xi_{\mathrm{can}}$ on $Y$ is obtained by Legendrian realizing each unknot with maximum possible rotation number imposed by its surgery coefficient. 
Proof. Suppose that $\left(Y, \xi_{\text {can }}\right)$ is diffeomorphic to the link of the rational surface singularity $(X, x)$. Then the minimal resolution $\pi: \tilde{X} \rightarrow X$ provides a holomorphic filling $(W, J)$ of $\left(Y, \xi_{\text {can }}\right)$. In particular, $W$ is a regular neighborhood of the exceptional divisor $E=\bigcup E_{j}$ of $\pi$. Since the curves $E_{j}$ are holomorphic, by the adjunction formula, we have

$$
\left\langle c_{1}(J),\left[E_{j}\right]\right\rangle=E_{j} \cdot E_{j}-2 \operatorname{genus}\left(E_{j}\right)+2=E_{j} \cdot E_{j}+2 .
$$

Recall that in Section 6 we discussed the classification of tight contact structures on a small fibered 3-manifold $Y$ under the assumption that $Y$ is an $L$-space for the case $e_{0}=-2$, which is satisfied for a rational singularity link. For each such 3-manifold $Y$, there are finitely many tight contact structures $\xi_{1}, \ldots, \xi_{m}$, all of which are Stein fillable. Moreover, for $i=1, \ldots, m$, a Stein filling $\left(W^{i}, J^{i}\right)$ of $\xi_{i}$ is given by taking a Legendrian surgery diagram, obtained from the plumbing tree describing $Y$, with the zigzags chosen in a certain way. Denote by $U_{j}^{i}$ a component of the corresponding Legendrian link and let $S_{j}^{i}$ denote the associated surface in the Stein filling $\left(W^{i}, J^{i}\right)$ obtained by pushing a Seifert surface for $U_{j}^{i}$ into the 4-ball and capping off by the core of the corresponding 2-handle (see [Gompf 1998]). Each $W^{i}$ is diffeomorphic to $W$ by a diffeomorphism which carries $S_{j}^{i}$ to $E_{j}$ for each $j$.

Now, using the well-known identities

$$
S_{j}^{i} \cdot S_{j}^{i}=\operatorname{tb}\left(U_{j}^{i}\right)-1, \quad\left\langle c_{1}\left(J^{i}\right),\left[S_{j}^{i}\right]\right\rangle=\operatorname{rot}\left(U_{j}^{i}\right)
$$

(see [Gompf 1998] for the second), observe that $\left\langle c_{1}\left(J^{i}\right),\left[S_{j}^{i}\right]\right\rangle=S_{j}^{i} \cdot S_{j}^{i}+2$ precisely when $\operatorname{rot}\left(U_{j}^{i}\right)=\operatorname{tb}\left(U_{j}^{i}\right)+1$. Since the latter equality holds exactly when all the cusps of $U_{j}^{i}$ except one are up cusps, it follows that $\left\langle c_{1}(J),\left[E_{j}\right]\right\rangle=\left\langle c_{1}\left(J^{i}\right),\left[S_{j}^{i}\right]\right\rangle$ for each $j$ precisely when all the extra zigzags are chosen so the additional cusps are all up cusps, that is, when all the extra zigzags are chosen on the same fixed side (which is determined by the orientation of the Legendrian unknots). In the finite list of tight contact structures on $Y$ there is only one such Stein fillable contact structure up to isomorphism [Wu 2006; Ghiggini 2008], which completes the proof.

\section{Milnor versus support genus}

In this section, we describe an infinite family of Milnor fillable contact 3-manifolds whose canonical contact structure has support genus (respectively norm) strictly less than its Milnor genus (respectively norm).

Consider the small Seifert fibered 3-manifold

$$
Y_{p}=Y\left(-2 ; \frac{1}{3}, \frac{2}{3}, \frac{p}{p+1}\right)
$$




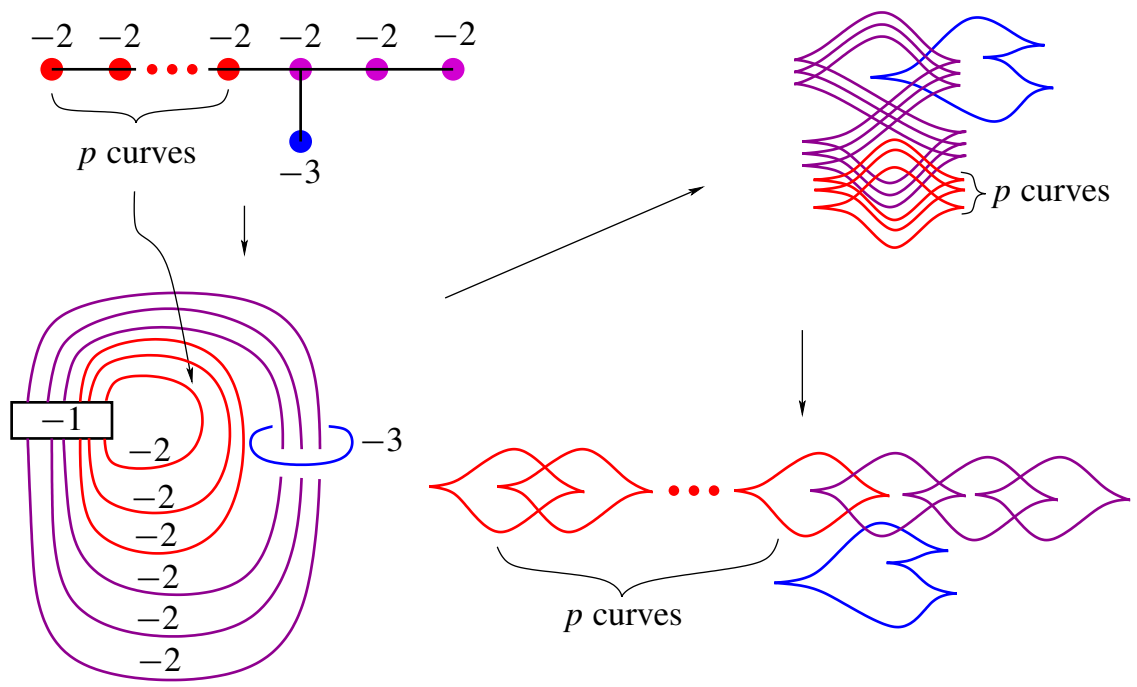

Figure 2. The contact structure $\xi \cong \xi_{1} \cong \xi_{\text {can }}$ on $Y_{p}$.

for $p \geq 2$. Observe that $Y_{p}$, whose dual resolution graph $\Gamma_{p}$ is shown in Figure 2, is the link of a rational complex surface singularity. By the classification of the tight contact structures on $Y_{p}$ given in [Ghiggini 2008], there are exactly two nonisotopic tight contact structures $\xi_{1}$ and $\xi_{2}$ on $Y_{p}$, both of which are Stein fillable. A Legendrian surgery diagram of $\xi_{1}$ is depicted on the bottom right in Figure 2. By putting the extra zigzag on the blue curve on the opposite side, we get a diagram for $\xi_{2}$.

Proposition 9.1. For $i=1,2$, we have $\operatorname{sg}\left(\xi_{i}\right) \leq 1$ and $\operatorname{sn}\left(\xi_{i}\right)=2$.

Proof. We first construct a supporting elliptic (that is, genus one) open book with two binding components for some Stein fillable contact structure $\xi$ on $Y_{p}$ following the recipe in [Etnyre and Ozbagci 2006]. Start from the plumbing diagram on the top left in Figure 2 (which is equivalent to a smooth surgery diagram including only unknots linked according to the given tree) and "roll up" this diagram by appropriately sliding handles to obtain the surgery diagram of $Y_{p}$ on the bottom left. Next, Legendrian realize the given surgery curves as depicted on the top right in Figure 2 to obtain the Legendrian surgery diagram for some Stein fillable contact structure $\xi$ on $Y_{p}$, which is isomorphic to $\xi_{1}$ depicted on the bottom right. Refer to [Etnyre and Ozbagci 2006] for the justification of such statements.

In order to construct an open book of $Y_{p}$ supporting $\xi$, start from an open book of $S^{3}$ and then embed the surgery curves onto the pages as depicted on the left in Figure 3 (the colors make it easier to follow how the surgery curves are embedded on the page). The initial page is a torus with one boundary component, and the 


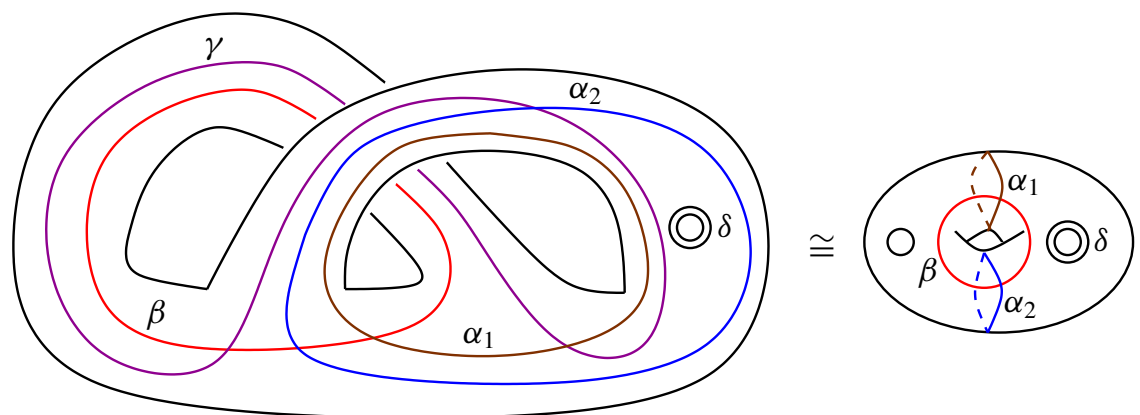

Figure 3. The page of an open book compatible with $\xi$.

monodromy of this open book of $S^{3}$ before the surgery is given by $\beta \alpha_{1}$, where $\alpha_{1}$ and $\beta$ generate the first homology group of the page. Now apply Legendrian surgeries along the given curves to get an open book of $Y_{p}$ with monodromy $\phi_{p}=\alpha_{2} \gamma^{3} \beta^{p} \beta \alpha_{1} \delta$, where $\delta$ is parallel to the small puncture on the torus, which occurs as a result of stabilizing the page appropriately. Next move $\beta$ over $\gamma^{3}$ to the left and use the fact that $\gamma \beta=\beta \alpha_{1}$ to get $\phi_{p}=\alpha_{2} \beta \alpha_{1}^{3} \beta^{p} \alpha_{1} \delta$. Then use the well-known braid relations and some simple overall conjugations to obtain a more symmetrical presentation of the monodromy as

$$
\phi_{p}=\left(\alpha_{2} \beta\right)^{2}\left(\alpha_{1} \beta\right)^{2} \beta^{p-2} \delta .
$$

This describes an abstract open book which is compatible with $\xi$, where the page is a torus with two boundary components and monodromy is $\phi_{p}$. Note that $\xi$ is isomorphic to $\xi_{1}$ (which is isomorphic to $\xi_{2}$, since one cannot distinguish the abstract open books corresponding to $\xi_{1}$ and $\xi_{2}$ ). It follows that $\operatorname{sg}\left(\xi_{i}\right) \leq 1$, since we have already constructed a genus one open book compatible with $\xi$.

Using the handlebody diagram of $Y_{p}$ depicted on the bottom left in Figure 2, it is straightforward to calculate that

$$
H_{1}\left(Y_{p}, \mathbb{Z}\right)= \begin{cases}\mathbb{Z}_{3} \oplus \mathbb{Z}_{3} & p=2 \bmod 3, \\ \mathbb{Z}_{9} & \text { otherwise. }\end{cases}
$$

Moreover, one can show that the Poincaré dual $\operatorname{PD}\left(e\left(\xi_{i}\right)\right) \in H_{1}\left(Y_{p}, \mathbb{Z}\right)$ of the Euler class $e\left(\xi_{i}\right)$ is a generator of one of the $\mathbb{Z}_{3}$-factors when $p$ is congruent to $2 \bmod 3$. Similarly $\operatorname{PD}\left(e\left(\xi_{i}\right)\right)$ is a generator of $H_{1}\left(Y_{p}, \mathbb{Z}\right)$ when $p$ is not congruent to $2 \mathrm{mod}$ 3 . Therefore the contact structure $\xi_{i}$ cannot be compatible with an elliptic open book with connected binding by [Etnyre and Ozbagci 2008, Lemma 6.1], since $e\left(\xi_{i}\right) \neq 0$. Note that $e\left(\xi_{1}\right)=-e\left(\xi_{2}\right)$, which implies that $\xi_{1}$ is not homotopic to $\xi_{2}$ as oriented plane fields, although they are isomorphic to each other. In fact, $\xi_{2}$ is obtained from $\xi_{1}$ by reversing the orientation of the underlying plane field. 
Now we claim that $\operatorname{sn}\left(\xi_{i}\right)=2$. To prove this, we need to exclude the possibility that $\xi_{i}$ is compatible with a planar open book with less than four binding components. Suppose that $\xi_{i}$ is compatible with a planar open book, that is, $\operatorname{sg}\left(\xi_{i}\right)=0$. If $\operatorname{bn}\left(\xi_{i}\right) \leq 2$, then $\xi_{i}$ is the unique tight contact structure on the lens space $L(n, n-1)$ for some $n \geq 0$ (see [Etnyre and Ozbagci 2008]) which is indeed impossible since $Y_{p}$ is not a lens space.

Next we rule out the possibility that $\operatorname{bn}\left(\xi_{i}\right)=3$. Let $\Sigma$ be the planar surface with three boundary components. Any diffeomorphism of $\Sigma$ is determined by three numbers $q, r, s$ that give the number of Dehn twists on curves $\tau_{1}, \tau_{2}, \tau_{3}$ parallel to each boundary component. It is easy to see that the 3-manifold determined by the open book with page $\Sigma$ and monodromy given by $\tau_{1}^{q} \tau_{2}^{r} \tau_{3}^{s}$ is the Seifert fibered 3-manifold $Y\left(0,-\frac{1}{q},-\frac{1}{r},-\frac{1}{s}\right)$. The first homology group of $Y\left(0,-\frac{1}{q},-\frac{1}{r},-\frac{1}{s}\right)$ has order $q r+q s+r s$.

Suppose that $\xi_{i}$ is compatible with an open book with page $\Sigma$ and monodromy $\tau_{1}^{q} \tau_{2}^{r} \tau_{3}^{s}$. The tightness of $\xi_{i}$ implies that the integers $q, r$, and $s$ are all nonnegative, because otherwise $\tau_{1}^{q} \tau_{2}^{r} \tau_{3}^{s}$ is not right-veering [Honda et al. 2007]. Moreover, since the order of the first homology group of $Y_{p}$ is 9 , for all $p \geq 2$, we conclude that $(q, r, s)$ is equal to either $(0,1,9),(0,3,3)$ or $(1,1,4)$. Hence $Y_{p}$ is diffeomorphic to either $L(9,8), L(3,2) \# L(3,2)$ or $L(9,4)$, which is a contradiction. Hence, $\operatorname{bn}\left(\xi_{i}\right) \geq 4$. This finishes the proof of our claim that $\operatorname{sn}\left(\xi_{i}\right)=2$.

One can ask whether or not $\operatorname{sg}\left(\xi_{i}\right)=1$, although it is not essential for the purposes of this paper. There are two known methods for finding obstructions to the planarity of a contact structure; see [Etnyre 2004; Ozsváth et al. 2005]. Unfortunately, both fail in our case, because $Y_{p}$ is an $L$-space and it is not an integral homology sphere.

Proposition 9.2. For the canonical contact structure $\xi_{\mathrm{can}}$ on the rational singularity link $Y_{p}$, we have $\operatorname{Mg}\left(\xi_{\text {can }}\right)=2$ and $\operatorname{Mn}\left(\xi_{\text {can }}\right)=3$.

Proof. Enumerate the vertices of the plumbing graph of $Y_{p}$ depicted in Figure 2 from left to right along the top row with the bottom vertex coming last. It is then easy to check that the $(p+4)$-tuple of positive integers $\underline{m}$ corresponding to the fundamental cycle of the minimal resolution of the singularity of which $Y_{p}$ is the link is given by $\underline{m}=(1,2,3,3, \ldots, 3,3,2,1,1)$. The construction in [Bhupal 2009] now gives an open book decomposition $\mathscr{O} \mathscr{B}(\underline{m})=\mathscr{O}^{\mathrm{min}}$ of $Y_{p}$ with binding a vertical link of type $\underline{n}=(0,0,1,0, \ldots, 0)$, where $\underline{m}$ and $\underline{n}$ are related by

$$
I\left(\Gamma_{p}\right) \underline{m}^{t}=-\underline{n}^{t} .
$$

Using formula (5-2) with $r=p+4$ gives $\operatorname{Mg}\left(\xi_{\text {can }}\right)=g(\mathscr{O B}(\underline{m}))=2$ for the canonical contact structure $\xi_{\text {can }}$ on $Y_{p}$. Also, $\mathrm{Mb}\left(\xi_{\text {can }}\right)=\operatorname{bc}(\mathscr{C} \mathscr{B}(\underline{m}))=\sum_{i=1}^{p+4} n_{i}=1$ and therefore $\operatorname{Mn}\left(\xi_{\text {can }}\right)=3$. 


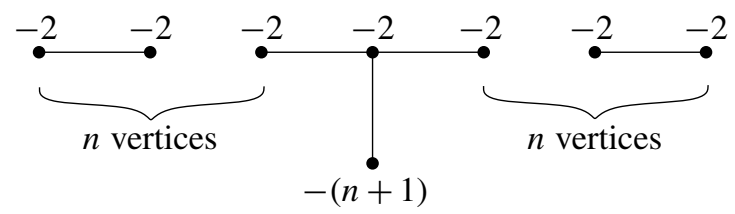

Figure 4. The plumbing graph for $P_{n}$.

Corollary 9.3. For $p \geq 2$, we have $\operatorname{sg}\left(\xi_{\text {can }}\right)<\operatorname{Mg}\left(\xi_{\text {can }}\right)$ and $\operatorname{sn}\left(\xi_{\text {can }}\right)<\operatorname{Mn}\left(\xi_{\text {can }}\right)$ for the canonical contact structure $\xi_{\mathrm{can}}$ on the singularity link $Y_{p}$.

Proof. Since any Milnor fillable contact structure is Stein fillable, $\xi_{\text {can }}$ is isomorphic to $\xi_{i}$ by Ghiggini's classification [2008]. (It does not make sense to distinguish $\xi_{1}$ and $\xi_{2}$ here since they are isomorphic to each other.) Thus Proposition 9.1 coupled with Proposition 9.2 clearly implies the corollary.

Remark 9.4. In contrast, $\operatorname{Mb}\left(\xi_{\text {can }}\right)=1$ while bn $\left(\xi_{\text {can }}\right) \geq 2$, which shows that the binding number is not necessarily less than or equal to the Milnor binding number.

We can improve Corollary 9.3 as follows.

Theorem 9.5. For each positive integer $k$, there exists a Milnor fillable contact 3-manifold such that $\operatorname{Mg}\left(\xi_{\mathrm{can}}\right)-\operatorname{sg}\left(\xi_{\mathrm{can}}\right) \geq k$ and $\operatorname{Mn}\left(\xi_{\mathrm{can}}\right)-\operatorname{sn}\left(\xi_{\mathrm{can}}\right) \geq k$.

Proof. The small Seifert fibered 3-manifold $P_{n}=Y\left(-2 ; \frac{1}{n+1}, \frac{n}{n+1}, \frac{n}{n+1}\right)$ for $n \geq 2$ is a rational singularity link, whose dual resolution graph is depicted in Figure 4. There are exactly $n$ nonisotopic tight contact structures $\xi_{1}, \ldots, \xi_{n}$ on $P_{n}$, each of which is Stein fillable [Ghiggini 2008]. On the other hand, $\xi_{i}$ is supported by an elliptic open book with $n$ binding components for $1 \leq i \leq n$ [Etnyre and Ozbagci 2006]. This proves that $\operatorname{sg}\left(\xi_{\text {can }}\right) \leq 1$ and $\operatorname{sn}\left(\xi_{\text {can }}\right) \leq n$ since the canonical contact structure $\xi_{\text {can }}$ on $P_{n}$ is isomorphic to $\xi_{i}$ for some $1 \leq i \leq n$.

Now enumerate the vertices of the graph in Figure 4 from left to right along the top row with the bottom vertex coming last and consider the $(2 n+2)$-tuple $\underline{m}=(1,2,3, \ldots, n-1, n, n+1, n, n-1, \ldots, 3,2,1,1)$ of positive integers. This corresponds to the fundamental cycle of the minimal resolution of the singularity of which $P_{n}$ is the link. It follows that $\operatorname{Mg}\left(\xi_{\text {can }}\right)=n$ and $\operatorname{Mn}\left(\xi_{\text {can }}\right)=2 n-1$. Taking $k=n-1$ now proves the theorem.

\section{Final remarks}

The minimal Milnor open book $\mathscr{O S}_{\min }$ on $Y=Y\left(e_{0} ; r_{1}, r_{2}, r_{3}\right)$ realizes $\operatorname{Mg}\left(\xi_{\text {can }}\right)$, $\mathrm{Mb}\left(\xi_{\mathrm{can}}\right)$ and $\mathrm{Mn}\left(\xi_{\mathrm{can}}\right)$. In fact, it follows from the proof of Theorem 5.1 given in [Altınok and Bhupal 2008] that $\mathscr{O}^{\circ}{ }_{\min }$ is the unique Milnor open book that realizes $\operatorname{Mg}\left(\xi_{\text {can }}\right), \operatorname{Mb}\left(\xi_{\text {can }}\right)$ and $\operatorname{Mn}\left(\xi_{\text {can }}\right)$. Thus any other Milnor open book on $Y$ that realizes $\operatorname{Mg}\left(\xi_{\text {can }}\right)$ cannot realize $\operatorname{Mb}\left(\xi_{\text {can }}\right)$ and $\operatorname{Mn}\left(\xi_{\text {can }}\right)$. For example, consider 

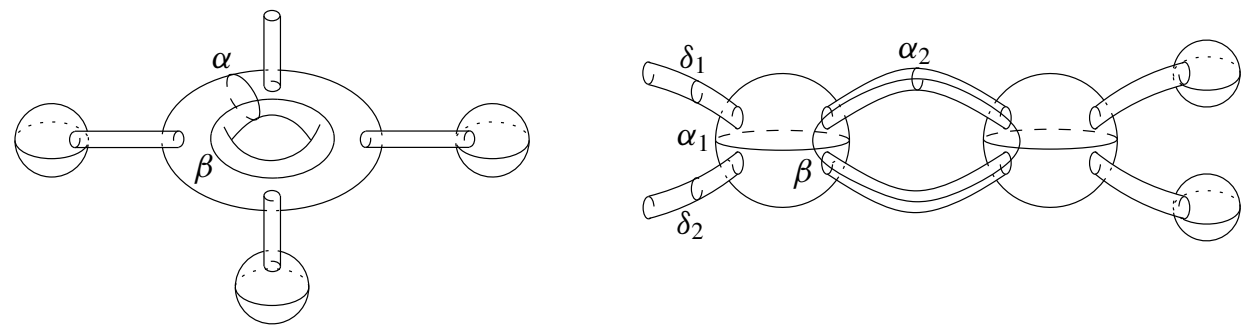

Figure 5. Pages of two different Milnor open books on $Y\left(-2 ; \frac{1}{2}, \frac{1}{2}, \frac{1}{2}\right)$.

the 3-manifold $Y=Y\left(-2 ; \frac{1}{2}, \frac{1}{2}, \frac{1}{2}\right)$, which is the link of the singularity $D_{4}$. The pages of two Milnor open books on $Y$ are given in Figure 5. The left pictures the minimal Milnor open book $\mathscr{O}_{\mathscr{B}_{\text {min }}}=\mathscr{O} \mathscr{S}_{(}((1,2,1,1))$ with page a once-punctured torus and monodromy $\phi=(\alpha \beta)^{3}$; the right pictures the Milnor open book $\bigcirc \mathscr{B}=$ $\mathscr{O} \mathscr{B}((2,2,1,1))$ with page a twice-punctured torus and monodromy $\psi$ satisfying $\psi^{2}=\delta_{1} \delta_{2} \alpha_{2}^{2}$. Using the uniqueness result from [Bonatti and Paris 2009] and the two-holed torus relation, one can check that $\psi=\alpha_{1} \alpha_{2} \beta \alpha_{2}^{2} \beta \alpha_{2}$. It is easy to see that $\mathscr{O} \mathscr{B}$ is related to $\mathscr{O}_{B_{\text {min }}}$ by a single positive stabilization.

\section{Acknowledgements}

We would like to thank András I. Stipsicz, John B. Etnyre and especially the referee for suggesting numerous improvements after reading earlier versions of this article. The second author would also like to thank the Mathematical Sciences Research Institute for its hospitality during the Symplectic and Contact Geometry and Topology program 009/10.

\section{References}

[Altınok and Bhupal 2008] S. Altınok and M. Bhupal, "Minimal page-genus of Milnor open books on links of rational surface singularities", pp. 1-10 in Singularities II, edited by J.-P. Brasselet et al., Contemp. Math. 475, Amer. Math. Soc., Providence, RI, 2008. MR 2010d:32030 Zbl 1195.57057

[Artin 1966] M. Artin, "On isolated rational singularities of surfaces", Amer. J. Math. 88 (1966), 129-136. MR 33 \#7340 Zbl 0142.18602

[Baldwin and Etnyre 2011] J. A. Baldwin and J. B. Etnyre, "A note on the support norm of a contact structure", pp. 79-88 in Low-dimensional and symplectic topology (Athens, GA, 2009), edited by M. Usher, Proc. Symp. Pure. Math. 82, Amer. Math. Soc., Providence, RI, 2011. Zbl 05995067

[Bhupal 2009] M. Bhupal, "Open book decompositions of links of simple surface singularities", Internat. J. Math. 20:12 (2009), 1527-1545. MR 2011e:57041 Zbl 1195.57058

[Bogomolov and de Oliveira 1997] F. A. Bogomolov and B. de Oliveira, "Stein small deformations of strictly pseudoconvex surfaces", pp. 25-41 in Birational algebraic geometry (Baltimore, MD, 1996), edited by Y. Kawamata and V. V. Shokurov, Contemp. Math. 207, Amer. Math. Soc., Providence, RI, 1997. MR 99a:32015 Zbl 0889.32021 
[Bonatti and Paris 2009] C. Bonatti and L. Paris, "Roots in the mapping class groups", Proc. Lond. Math. Soc. (3) 98:2 (2009), 471-503. MR 2010a:57038 Zbl 1160.57014

[Caubel and Popescu-Pampu 2004] C. Caubel and P. Popescu-Pampu, "On the contact boundaries of normal surface singularities”, C. R. Math. Acad. Sci. Paris 339:1 (2004), 43-48. MR 2005e:32046 Zbl 1080.32025

[Caubel et al. 2006] C. Caubel, A. Némethi, and P. Popescu-Pampu, "Milnor open books and Milnor fillable contact 3-manifolds”, Topology 45:3 (2006), 673-689. MR 2007a:53158 Zbl 1098.53064

[Ding and Geiges 2004] F. Ding and H. Geiges, "A Legendrian surgery presentation of contact 3-manifolds", Math. Proc. Cambridge Philos. Soc. 136:3 (2004), 583-598. MR 2005m:57038 Zbl 1069.57015

[Eliashberg 1990] Y. Eliashberg, "Topological characterization of Stein manifolds of dimension >2", Internat. J. Math. 1:1 (1990), 29-46. MR 91k:32012 Zbl 0699.58002

[Eliashberg and Gromov 1991] Y. Eliashberg and M. Gromov, "Convex symplectic manifolds", pp. 135-162 in Several complex variables and complex geometry, Part 2 (Santa Cruz, CA, 1989), edited by E. Bedford et al., Proc. Sympos. Pure Math. 52, Amer. Math. Soc., Providence, RI, 1991. MR 93f:58073 Zbl 0742.53010

[Etgü and Lekili 2010] T. Etgü and Y. Lekili, "Examples of planar tight contact structures with support norm one", Int. Math. Res. Not. 2010:19 (2010), 3723-3728. MR 2011j:57034 Zbl 1201.57018

[Etgü and Ozbagci 2006] T. Etgü and B. Ozbagci, "Explicit horizontal open books on some plumbings", Internat. J. Math. 17:9 (2006), 1013-1031. MR 2007j:57028 Zbl 1114.57030

[Etnyre 2004] J. B. Etnyre, "Planar open book decompositions and contact structures", Int. Math. Res. Not. 2004:79 (2004), 4255-4267. MR 2005k:57049 Zbl 1069.57016

[Etnyre 2006] J. B. Etnyre, "Lectures on open book decompositions and contact structures", pp. 103-141 in Floer homology, gauge theory, and low-dimensional topology, edited by D. A. Ellwood et al., Clay Math. Proc. 5, Amer. Math. Soc., Providence, RI, 2006. MR 2007g:57042 Zbl 1069.57016

[Etnyre and Ozbagci 2006] J. B. Etnyre and B. Ozbagci, "Open books and plumbings", Int. Math. Res. Not. 2006 (2006), Art. ID 72710, 17. MR 2007k:57050

[Etnyre and Ozbagci 2008] J. B. Etnyre and B. Ozbagci, "Invariants of contact structures from open books", Trans. Amer. Math. Soc. 360:6 (2008), 3133-3151. MR 2009g:57041 Zbl 1157.57015

[Geiges 2008] H. Geiges, An introduction to contact topology, Cambridge Studies in Advanced Mathematics 109, Cambridge University Press, 2008. MR 2008m:57064 Zbl 1153.53002

[Ghiggini 2008] P. Ghiggini, "On tight contact structures with negative maximal twisting number on small Seifert manifolds", Algebr. Geom. Topol. 8:1 (2008), 381-396. MR 2009f:57038 Zbl 1148.57026

[Giroux 2002] E. Giroux, "Géométrie de contact: de la dimension trois vers les dimensions supérieures”, pp. 405-414 in Proceedings of the International Congress of Mathematicians, Vol. II (Beijing, 2002), edited by T. Li, Higher Ed. Press, Beijing, 2002. MR 2004c:53144 Zbl 1015.53049

[Gompf 1998] R. E. Gompf, "Handlebody construction of Stein surfaces", Ann. of Math. (2) 148:2 (1998), 619-693. MR 2000a:57070 Zbl 0919.57012

[Grauert 1962] H. Grauert, "Über Modifikationen und exzeptionelle analytische Mengen", Math. Ann. 146 (1962), 331-368. MR 25 \#583 Zbl 0178.42702

[Honda et al. 2007] K. Honda, W. H. Kazez, and G. Matić, "Right-veering diffeomorphisms of compact surfaces with boundary”, Invent. Math. 169:2 (2007), 427-449. MR 2008e:57028 Zbl 1167.57008 
[Laufer 1972] H. B. Laufer, "On rational singularities”, Amer. J. Math. 94 (1972), 597-608. MR 48 \#8837 Zbl 0251.32002

[Lekili and Ozbagci 2010] Y. Lekili and B. Ozbagci, "Milnor fillable contact structures are universally tight”, Math. Res. Lett. 17:6 (2010), 1055-1063. MR 2011m:57033 Zbl 1222.53083

[Milnor 1968] J. Milnor, Singular points of complex hypersurfaces, Annals of Mathematics Studies 61, Princeton University Press, 1968. MR 39 \#969 Zbl 0184.48405

[Mumford 1961] D. Mumford, "The topology of normal singularities of an algebraic surface and a criterion for simplicity", Inst. Hautes Études Sci. Publ. Math. 9 (1961), 5-22. MR 27 \#3643 Zbl 0108.16801

[Némethi 1999] A. Némethi, "Five lectures on normal surface singularities", pp. 269-351 in Low dimensional topology (Eger, 1996/Budapest, 1998), edited by K. Böröczky, Jr. et al., Bolyai Soc. Math. Stud. 8, János Bolyai Math. Soc., Budapest, 1999. MR 2001g:32066 Zbl 0958.32026

[Némethi 2005] A. Némethi, "On the Ozsváth-Szabó invariant of negative definite plumbed 3 manifolds”, Geom. Topol. 9 (2005), 991-1042. MR 2006c:57011 Zbl 1138.57301

[Némethi 2008] A. Némethi, "On the canonical contact structure of links of complex surface singularities", preprint, 2008, available at http://tinyurl.com/7hfkmml.

[Némethi and Tosun 2011] A. Némethi and M. Tosun, "Invariants of open books of links of surface singularities”, Studia Sci. Mat. Hungarica 48:1 (2011), 135-144.

[Ozbagci 2008] B. Ozbagci, "Surgery diagrams for horizontal contact structures", Acta Math. Hungar. 120:1-2 (2008), 193-208. MR 2009e:57047 Zbl 1174.57001

[Ozsváth et al. 2005] P. Ozsváth, A. Stipsicz, and Z. Szabó, "Planar open books and Floer homology”, Int. Math. Res. Not. 2005:54 (2005), 3385-3401. MR 2006j:57056 Zbl 1092.57014

[Thurston and Winkelnkemper 1975] W. P. Thurston and H. E. Winkelnkemper, "On the existence of contact forms", Proc. Amer. Math. Soc. 52 (1975), 345-347. MR 51 \#11561 Zbl 0312.53028

[Weinstein 1991] A. Weinstein, "Contact surgery and symplectic handlebodies", Hokkaido Math. J. 20:2 (1991), 241-251. MR 92g:53028 Zbl 0737.57012

[Wu 2006] H. Wu, "Legendrian vertical circles in small Seifert spaces", Commun. Contemp. Math. 8:2 (2006), 219-246. MR 2007f:57053 Zbl 1104.57015

Received December 10, 2010. Revised August 18, 2011.

MOHAN BHUPAL

DEPARTMENT OF MATHEMATICS

Middle East TeChNiCAL UNIVERSity

06531 ANKARA

TURKEY

bhupal@metu.edu.tr

http://www.metu.edu.tr/ bhupal/

BURAK OZBAGCI

DEPARTMENT OF MATHEMATICS

KOÇ UNIVERSITY

34450 ISTANBUL

TURKEY

bozbagci@ku.edu.tr

http://home.ku.edu.tr/ bozbagci/ 


\title{
PACIFIC JOURNAL OF MATHEMATICS
}

\author{
http://pacificmath.org \\ Founded in 1951 by \\ E. F. Beckenbach (1906-1982) and F. Wolf (1904-1989)
}

\section{EDITORS}

V. S. Varadarajan (Managing Editor)

Department of Mathematics

University of California

Los Angeles, CA 90095-1555

pacific@math.ucla.edu

Vyjayanthi Chari

Department of Mathematics

University of California

Riverside, CA 92521-0135

chari@math.ucr.edu

\section{Robert Finn}

Department of Mathematics Stanford University

Stanford, CA 94305-2125

finn@math.stanford.edu

Kefeng Liu

Department of Mathematics

University of California

Los Angeles, CA 90095-1555

liu@math.ucla.edu
Darren Long

Department of Mathematics

University of California

Santa Barbara, CA 93106-3080

long@math.ucsb.edu

Jiang-Hua Lu

Department of Mathematics

The University of Hong Kong

Pokfulam Rd., Hong Kong jhlu@maths.hku.hk

Alexander Merkurjev

Department of Mathematics

University of California

Los Angeles, CA 90095-1555

merkurev@math.ucla.edu
Sorin Popa

Department of Mathematics University of California

Los Angeles, CA 90095-1555 popa@math.ucla.edu

Jie Qing

Department of Mathematics

University of California

Santa Cruz, CA 95064

qing@cats.ucsc.edu

Jonathan Rogawski

Department of Mathematics

University of California

Los Angeles, CA 90095-1555

jonr@math.ucla.edu

\section{PRODUCTION}

pacific@math.berkeley.edu

\section{SUPPORTING INSTITUTIONS}

ACADEMIA SINICA, TAIPEI

CALIFORNIA INST. OF TECHNOLOGY INST. DE MATEMÁTICA PURA E APLICADA KEIO UNIVERSITY

MATH. SCIENCES RESEARCH INSTITUTE NEW MEXICO STATE UNIV.

OREGON STATE UNIV.

\author{
STANFORD UNIVERSITY \\ UNIV. OF BRITISH COLUMBIA \\ UNIV. OF CALIFORNIA, BERKELEY \\ UNIV. OF CALIFORNIA, DAVIS \\ UNIV. OF CALIFORNIA, LOS ANGELES \\ UNIV. OF CALIFORNIA, RIVERSIDE \\ UNIV. OF CALIFORNIA, SAN DIEGO \\ UNIV. OF CALIF., SANTA BARBARA
}

\author{
UNIV. OF CALIF., SANTA CRUZ \\ UNIV. OF MONTANA \\ UNIV. OF OREGON \\ UNIV. OF SOUTHERN CALIFORNIA \\ UNIV. OF UTAH \\ UNIV. OF WASHINGTON \\ WASHINGTON STATE UNIVERSITY
}

These supporting institutions contribute to the cost of publication of this Journal, but they are not owners or publishers and have no responsibility for its contents or policies.

See inside back cover or pacificmath.org for submission instructions.

The subscription price for 2011 is US \$420/year for the electronic version, and \$485/year for print and electronic.

Subscriptions, requests for back issues from the last three years and changes of subscribers address should be sent to Pacific Journal of Mathematics, P.O. Box 4163, Berkeley, CA 94704-0163, U.S.A. Prior back issues are obtainable from Periodicals Service Company, 11 Main Street, Germantown, NY 12526-5635. The Pacific Journal of Mathematics is indexed by Mathematical Reviews, Zentralblatt MATH, PASCAL CNRS Index, Referativnyi Zhurnal, Current Mathematical Publications and the Science Citation Index.

The Pacific Journal of Mathematics (ISSN 0030-8730) at the University of California, c/o Department of Mathematics, 969 Evans Hall, Berkeley, CA 94720-3840, is published monthly except July and August. Periodical rate postage paid at Berkeley, CA 94704, and additional mailing offices. POSTMASTER: send address changes to Pacific Journal of Mathematics, P.O. Box 4163, Berkeley, CA 94704-0163.

PJM peer review and production are managed by EditFLOW ${ }^{\mathrm{TM}}$ from Mathematical Sciences Publishers.

PUBLISHED BY PACIFIC JOURNAL OF MATHEMATICS

at the University of California, Berkeley 94720-3840

A NON-PROFIT CORPORATION

Typeset in LATEX

Copyright $(2011$ by Pacific Journal of Mathematics 


\section{PACIFIC JOURNAL OF MATHEMATICS}

Volume $254 \quad$ No. $1 \quad$ November 2011

A mean curvature estimate for cylindrically bounded submanifolds

Luis J. AlíAS and MARCos DAJCZER

Weyl group multiple Dirichlet series of type $C$

JENNIFER BEINEKE, BENJAMIN BRUBAKER and SHARON FrECHETTE

Milnor open books of links of some rational surface singularities

MOHAN BHUPAL and BURAK OZBAGCI

Simple closed curves, word length, and nilpotent quotients of free groups

KHALID BOU-RABEE and ASAF HADARI

Strong submodules of almost projective modules

GÁBOR BRAUN and JAN TRLIFAJ

Interlacing log-concavity of the Boros-Moll polynomials

William Y. C. Chen, LARry X. W. WAng and ERnest X. W. Xia

Schwarzian norms and two-point distortion

Martin Chuaqui, Peter Duren, William Ma, Diego Mejía, David

MINDA and BRAD OSGOOD

The principle of stationary phase for the Fourier transform of $D$-modules

JIANGXUE FANG

Monotonicity and uniqueness of a 3D transonic shock solution in a conic nozzle with

variable end pressure

JUN LI, ZHOUPING XIN and HUICHENG YIN

Refined open noncommutative Donaldson-Thomas invariants for small crepant resolutions

KENTARO NAGAO

The Dirichlet problem for harmonic functions on compact sets

TONY L. PERKINS

Extension of an analytic disc and domains in $\mathbb{C}^{2}$ with noncompact automorphism group

\section{MINJU SONG}

Regularity of the first eigenvalue of the $p$-Laplacian and Yamabe invariant along geometric flows

ER-MIN WANG and YU ZHENG 